\title{
Covariance-constrained difference inversion of time-lapse electrical resistivity tomography data
}

\author{
Thomas Hermans ${ }^{1}$, Andreas Kemna ${ }^{2}$, and Frédéric Nguyen ${ }^{3}$
}

\begin{abstract}
Hydrogeophysics has become a major field of research in the past two decades, and time-lapse electrical resistivity tomography (ERT) is one of the most popular techniques to monitor passive and active processes in shallow subsurface reservoirs. Time-lapse inversion schemes have been developed to refine inversion results, but they mostly still rely on a spatial regularization procedure based on the standard smoothness constraint. We have applied a covariance-based regularization operator to the time-lapse ERT inverse problem. We first evaluated the method for surface and crosshole ERT with two synthetic cases and compared the results with the smoothness-constrained
\end{abstract}

inversion (SCI). These tests showed that the covariance-constrained inversion (CCI) better images the target in terms of shape and amplitude. Although more important in low-sensitivity zones, we have observed improvements everywhere in the tomograms. Those synthetic examples also show that an error made in the range or in the type of the variogram model had a limited impact on the resulting image, which still remained better than SCI. We then applied the method to cross-borehole ERT field data from a heat-tracing experiment, in which the comparison with direct measurements showed a strong improvement of the breakthrough curves retrieved from ERT. This method could be extended to the time dimension, which would allow the use of $\mathrm{CCI}$ in $4 \mathrm{D}$ inversion schemes.

\section{INTRODUCTION}

Hydrogeophysics has become a major field of research in the past two decades, and time-lapse monitoring is now commonly applied to study the dynamic changes of subsurface reservoirs (Binley et al., 2015). Among the available geophysical techniques, time-lapse electrical resistivity tomography (ERT) is one of the most popular methods (Singha et al., 2015). Given its sensitivity to numerous soil/rock properties, ERT has been applied in various contexts, such as salt-tracer experiments (Doetsch et al., 2012b; Robert et al., 2012), dynamics of infiltration and saturation in the vadose zone (Binley et al., 2002; Koestel et al., 2008), monitoring of permafrost (Krautblatter et al., 2010; Supper et al., 2014), interaction between surface and groundwater (Coscia et al., 2011), and more recently to $\mathrm{CO}_{2}$ sequestration (Carrigan et al., 2013) and heat-tracing experiments (Hermans et al., 2014; Arato et al., 2015).

In contrast to static ERT where spatial variations of bulk electrical resistivity are related to several specific properties (saturation, salin- ity, soil/rock type, temperature, etc.), temporal electrical resistivity changes can generally be linked to only one of these parameters or states (e.g., temperature in heat-tracing experiments). Consequently, the results may be directly related to the phenomenon of interest. In addition, time-lapse inversion schemes have been developed to refine inversion results. The different inversion scheme takes advantage of the repetition of measurements over time to eliminate the systematic error component of the data (LaBrecque and Yang, 2001; Kemna et al., 2002). The 4D inversion scheme (Kim et al., 2009; Karaoulis et al., 2011) inverts multiple data sets simultaneously including the time dimension in the inversion process. However, deterministic time-lapse inversion schemes still remain dependent on the spatial or spatiotemporal regularization procedure required to overcome the ill-posedness of the ERT inverse problem.

The standard spatial regularization method is the application of a smoothness constraint (de Groot-Hedlin and Constable, 1990). However, such a constraint is often not coherent with the geology. Thus, many alternatives have been developed in static imaging,

Manuscript received by the Editor 18 September 2015; revised manuscript received 9 May 2016; published online 18 July 2016.

${ }^{1}$ Formerly University of Liege, Department ArGEnCo, GEO 3 , Applied Geophysics, Liege, Belgium; presently Stanford University, Geological Sciences, Stanford, California, USA.E-mail: thomas.hermans@ulg.ac.be; thermans@stanford.edu.

${ }^{2}$ University of Bonn, Department of Geophysics, Steinmann Institute, Bonn, Germany. E-mail: kemna@geo.uni-bonn.de.

${ }^{3}$ University of Liege, Department ArGEnCo, GEO 3 , Applied Geophysics, Liege, Belgium. E-mail: f.nguyen@ulg.ac.be.

(C) 2016 Society of Exploration Geophysicists. All rights reserved. 
such as blocky inversion (Farquharson and Oldenburg, 1998), structural inversion (Kaipio et al., 1999; Doetsch et al., 2012a), minimum support (MS) and gradient support functionals (Portnaguine and Zhdanov, 1999; Blaschek et al., 2008), or other prior information incorporation methods (Caterina et al., 2014).

Despite the fact that regularization in a time-lapse mode is similar to a static mode, very few studies have investigated the use of alternative spatial regularization operators in time-lapse studies more adapted to the physics of the process (Ajo-Franklin et al., 2007; Doetsch et al., 2010; Fiandaca et al., 2015; Nguyen et al., 2016). Coupled hydrogeophysical inversion schemes are promising. They are directly expressed in terms of process physics and therefore do not require any regularization. However, the complexity of the subsurface, the dependence on the hydrogeological conceptual model, and the uncertainty of the involved petrophysical relationships may limit their use to homogeneous or simple cases (Hinnel et al., 2011) or be computationally demanding (Irving and Singha, 2010).

Therefore, the need for regularization functionals coherent with the process of interest is as important in time lapse as it is in static inversion. Very recently, Fiandaca et al. (2015) and Nguyen et al. (2016) apply the MS and minimum gradient-support (MGS) functionals to time-lapse ERT inversion to produce focused time-lapse changes. This is of particular interest to image preferential flow paths in fractured aquifers or preferential infiltration processes. The MGS and smoothness constraints can be seen as two end members from very sharp to very smooth constraints. However, even for dispersed processes, such as solute transport in tracer experiments, the smoothness constraint may be too extreme to produce reliable changes, generally leading to an underestimation of the maximum resistivity variation, whereas MS approaches require determining the desired value of the MS parameter. In such contexts, the application of a specific correlation length through covariance-based constraints may be useful (Yeh and Liu, 2000; Holliger et al., 2008). Some authors have also proposed building physically based regularization operators based on training data sets (Oware et al., 2013) or running several inversions with varying inversion parameters (Audebert et al., 2014) to better identify the zones affected by resistivity changes.

Model parameter covariance matrices used for geophysical inversion are introduced by Franklin (1970) and Tarantola and Valette (1982). Maurer et al. (1998) generalize the approach for 2D inversion and compare it with the smooth-model operator. They show that the method tends to favor a zero-structure model. This approach is later applied by several authors for static ERT (Linde et al., 2006; Hermans et al., 2012). Johnson et al. (2007) develop an alternative regularization to ensure that variogram statistics are imposed during the inversion by explicitly calculating the variogram of the model. They apply the approach to cross-borehole GPR data and later to ERT data (Johnson et al., 2012). Their method is truly stochastic because they generate an ensemble of solutions.

In time-lapse studies, Day-Lewis et al. (2002) develop an inversion procedure based on a spatiotemporal parameter covariance matrix to invert crosswell GPR data and test it on a synthetic case. Their procedure enables taking into account the data acquisition time in the inversion. They impose spatial and temporal correlation lengths in the inversion. Similarly, Rosas Carbajal et al. (2012) apply the algorithm of Linde et al. (2006) to synthetic time-lapse magnetotelluric data using various norms to produce more or less sharp models. Doetsch et al. (2010) also use the algorithm of Linde et al. (2006) to jointly invert time-lapse GPR and ERT field data. However, these studies did not systematically investigate the role of the imposed correlation length, nor quantified the improvement brought by the technique compared with standard inversions.

In this paper, we propose to apply a covariance-based constraint as regularization operator to the time-lapse ERT inverse problem, termed covariance-constrained inversion (CCI) hereafter. We first discuss the details of the implementation. Then, we illustrate the method with two synthetic examples. The first example uses surface ERT, in which we compare the results with the standard smoothness-constrained inversion (SCI). We analyze the role of the imposed correlation length and the varying resolution in the tomograms. Then, we apply the methodology to a cross-borehole ERT example to illustrate the role of the sill and type of the covariance model. Finally, we apply the technique in a field case study in which a heat-tracing experiment was monitored (Hermans et al., 2015b). We use direct measurements by several control piezometers to compare the results and assess their quality. The paper ends with discussion and conclusion.

\section{TIME-LAPSE COVARIANCE-BASED INVERSION}

Our methodology is based on the inversion procedure presented in Hermans et al. (2012) combined with the difference inversion scheme presented in Kemna et al. (2002), both implemented in the 2.5D ERT inversion code CRTomo (Kemna, 2000).

\section{Model parameter covariance matrix}

In the inversion procedure, the roughness matrix commonly used in SCI for regularization is replaced by the model parameter covariance matrix $\mathbf{C}_{\mathbf{m}}$. This matrix describes how model parameters are correlated according to their distance from each other. For stationary processes, it is linked to the variogram

$$
\gamma(h)=C_{m}(0)-C_{m}(h),
$$

where $C_{m}(0)$ is the sill of the variogram, equal to the variance of the parameter, and $h$ is the lag, i.e., the distance between parameters. The variogram can be computed using experimental data and then modeled using standard approaches, such as the Gaussian, exponential, or spherical models (for a description of geostatistical concepts, see Isaaks and Srivastava, 1989). In the case of anisotropy, two (or three) main ranges (e.g., horizontal and vertical ranges) can be used to model the generalized range in every direction assuming an ellipsoidal distribution of ranges (Chasseriau and Chouteau, 2003). The generalized range can be subsequently used to compute the value of the covariance (equation 1 ) for any combination of model parameters in the grid and build the parameter covariance matrix used during the inversion process.

Applications for static conditions (Linde et al., 2006; Hermans et al., 2012; Caterina et al., 2014) have shown that the use of a CCI has generally led to a strong improvement of the resulting image compared with SCI. This operator also smooths the distribution of model parameters through the covariance matrix, but to a level that can be controlled by the correlation length. 


\section{Difference inversion scheme}

In the difference inversion scheme, data and model parameters are expressed in terms of differences compared with an initial state called the background:

$$
\Delta \mathbf{m}=\mathbf{m}-\mathbf{m}_{\mathbf{0}}, \quad \Delta \mathbf{d}=\mathbf{d}-\mathbf{d}_{\mathbf{0}},
$$

where, in this work, $\mathbf{m}$ and $\mathbf{m}_{\mathbf{0}}$ are the log electrical resistivity of the current model and the background, respectively, and $\mathbf{d}$ and $\mathbf{d}_{\mathbf{0}}$ are the corresponding log resistance data. To be consistent with the formulation of the difference inversion scheme, the model parameter covariance matrix must also be expressed in terms of model differences $\Delta \mathbf{m}$. From now on, we will refer to it as the model difference covariance matrix $\mathbf{C}_{\Delta \mathrm{m}}$

Geostatistical concepts are often used in geophysics to describe subsurface parameter distributions. The use of geostatistical tools such as the variogram theoretically requires the assumption of stationarity (Isaaks and Srivastava, 1989). Even if stationarity cannot be demonstrated, it is generally accepted for structural heterogeneity (i.e., for the absolute value distribution of a given parameter). In time-lapse applications, however, parameter changes are associated with changing variables of a process, which differs from a structural property (Michalak and Shlomi, 2007). The rigorous application of geostatistical approaches would therefore require first validating the proposed model difference covariance. In the present application, we are instead interested in the ability of the covariance matrix to regulate the correlation length of the sought model changes (Hermans et al., 2012). We thus accept using the concept in situations, in which theoretical statistical assumptions are not strictly verified (Day-Lewis et al., 2002; Doetsch et al., 2010; Rosas Carbajal et al., 2012).

The objective function of the inverse problem can be expressed as

$$
\begin{aligned}
\phi_{\text {diff }}(\boldsymbol{\Delta} \mathbf{m})= & \left\|\mathbf{C}_{\mathbf{\Delta d}}^{-0.5}\left[\mathbf{\Delta d}-\left(f(\mathbf{m})-f\left(\mathbf{m}_{\mathbf{0}}\right)\right)\right]\right\|_{2}^{2} \\
& +\lambda\left\|\mathbf{C}_{\mathbf{\Delta} \mathbf{m}}^{-0.5} \mathbf{\Delta} \mathbf{m}\right\|_{2}^{2},
\end{aligned}
$$

where $\mathbf{C}_{\boldsymbol{\Delta d}}$ is the data "noise" difference covariance matrix determined from error estimates, and $f$ is the forward operator mapping the model parameters to the data. The parameter $\lambda$ is the regularization parameter that balances between the data misfit and the model functional. In CRTomo, $\lambda$ is optimized at each iteration through a line search to get the minimum root-mean-square value of the error-weighted data misfit $\left(\varepsilon_{\mathrm{rms}}\right)$ for the maximum possible value of $\lambda$. The line search step is reduced as the inversion converges (for technical details, see Kemna, 2000). When erms becomes smaller than one, $\lambda$ is increased to get the solution that fits the data to the assumed error level $\left(\varepsilon_{\mathrm{rms}}=1\right)$ (de Groot-Hedlin and Constable, 1990; Kemna, 2000). We applied this criterion for all inversions presented in this paper; i.e., all inversions for SCI and CCI are fitted to the same error level with the same optimization of $\lambda$, ensuring a fair comparison between results.

In the cases presented in this study, we use a constant value of $C_{\Delta m}(0)$ for all parameters. In consequence, this parameter only acts as a multiplication constant of $\mathbf{C}_{\boldsymbol{\Delta m}}$ in the inversion process and its effect is overcome by the optimization made on the regularization parameter $\lambda$ (equation 3). This is also observed by Hermans et al. (2012) and Rosas Carbajal et al. (2012) and will be illustrated for the second synthetic case. We invert for model parameter changes that can explain the variations observed in the data. It means that all inversions are relative to the background model $\mathbf{m}_{\mathbf{0}}$ that must be obtained previously by a static inversion. The data difference covariance matrix $\mathbf{C}_{\Delta \mathbf{d}}$ is computed using an assessment of the data error. For field data, we use reciprocal measurements to compute a linear model of the resistance error given the measured resistance (Slater et al., 2000)

$$
|e|=b+c R,
$$

where $e$ is the reciprocal error, $b$ is an absolute resistance error component, $c$ is a relative resistance error component, and $R$ is the mean measured resistance.

\section{SYNTHETIC STUDIES}

\section{Surface synthetic case}

\section{Background model}

The first synthetic benchmark is based on surface ERT measurements inspired by a field case (Hermans et al., 2015a). The background model mimics a heterogeneous alluvial aquifer with a distribution of electrical resistivity ranging from $50 \Omega \mathrm{m}$ in loam and clay, located mainly in the upper part of the model, to more than $300 \Omega \mathrm{m}$ in sand and gravel located in the bottom part of the aquifer. The alluvial deposits lie on a more resistive bedrock.

The simulated electrode array consists of 64 electrodes with $2 \mathrm{~m}$ spacing (total length of $126 \mathrm{~m}$ ). The simulated data correspond to a dipole-dipole protocol with the dipole length less than or equal to nine electrode spacings and the dipole spacing less than or equal to six. This leads, for background and time-lapse data sets, to 1015 resistance data.

Figure 1a shows the anisotropic SCI of the background. Although leading to slightly different inversion results, other background inversions (such as CCI) have a negligible influence on the time-lapse inversion results. For the sake of conciseness, we limit ourselves to the use of the model presented in Figure 1a as background model $\mathbf{m}_{\mathbf{0}}$ for subsequent time-lapse inversions.

\section{Time-lapse model}

The time-lapse model used in this example is displayed in Figure $1 b$, it is defined in terms of percentage change of resistivity with respect to the background shown in Figure 1a:

$$
\Delta m \%=\frac{\rho_{\mathrm{TL}, i}-\rho_{\mathrm{BG}, i}}{\rho_{\mathrm{BG}, i}} \times 100,
$$

where $\rho_{\mathrm{TL}, i}$ and $\rho_{\mathrm{BG}, i}$ are, respectively, the resistivity of the $i$ th model parameter of the time-lapse and background models. The time-lapse model represents a confined horizontal anomaly located in the center of the model. Its extension is $25 \mathrm{~m}$ in its upper and bottom parts and $33 \mathrm{~m}$ in the middle. The maximum change is located at $6.25 \mathrm{~m}$ depth and corresponds to a change of resistivity of $50 \%$. The change of resistivity decreases linearly from $-50 \%$ to $0 \%$ for the five adjacent layers ( $0.5 \mathrm{~m}$ thick), upward and downward. Thus, the total thickness of the anomaly is $4.5 \mathrm{~m}$. Note that the forward and inversion grids with regular blocks were extended laterally and at depth to limit the effect of boundary conditions. 
This model was built as an alternative to the classical block model, for which smoothing constraints are obviously inappropriate. Here, the model displays gradual changes so that sharp or blocky models are not desirable. We computed the vertical and horizontal variograms for this time-lapse model using the spatial distribution of time-lapse changes in the logarithm of electrical resistivity. The calculated horizontal and vertical ranges with the Gaussian variogram model are, respectively, 32 and $3.2 \mathrm{~m}$ (Figure 2a and $2 b$ ).

Noise was added to the data with two random components: an absolute resistance component uniformly distributed between -0.0005 and $0.0005 \Omega$ and a relative resistance component uniformly distributed between $-0.25 \%$ and $0.25 \%$ of the resistance (equation 4). This relatively low noise level was chosen considering that lower noise levels are expected for time-lapse studies using the difference inversion because any systematic error component is suppressed (equation 2). The choice of two uniformly distributed noise components is made to better mimic the noise observed on real data sets. However, it has to be noted that a uniform distribution is inconsistent with the choice of an $l_{2}$-norm for the calculation of the data misfit, which assumes a Gaussian distribution of the error. Still, this is often neglected in practice (Pidlisecky et al., 2007). In this case, the effect is expected to be similar for SCI and CCI.

In addition to visual comparison, we will compare the inversions based on their normalized time-lapse model misfit $\left(M M_{\mathrm{TL}}\right)$. It is calculated based on the change in logarithm of resistivity in the inversion results compared with the corresponding change in the true model:

$$
M M_{\mathrm{TL}}=\frac{1}{M M_{0}} \sqrt{\frac{\sum_{i=1}^{n}\left(\log \frac{\rho_{\mathrm{TL}, i}}{\rho_{\mathrm{BG}, i}}-\log \frac{\rho_{\mathrm{TL}, i}^{*}}{\rho_{\mathrm{BG}, i}^{*}}\right)^{2}}{n}},
$$

where * makes reference to the inverted models in comparison with the true models and $n$ is the number of cells in the grid. The $M M_{0}$
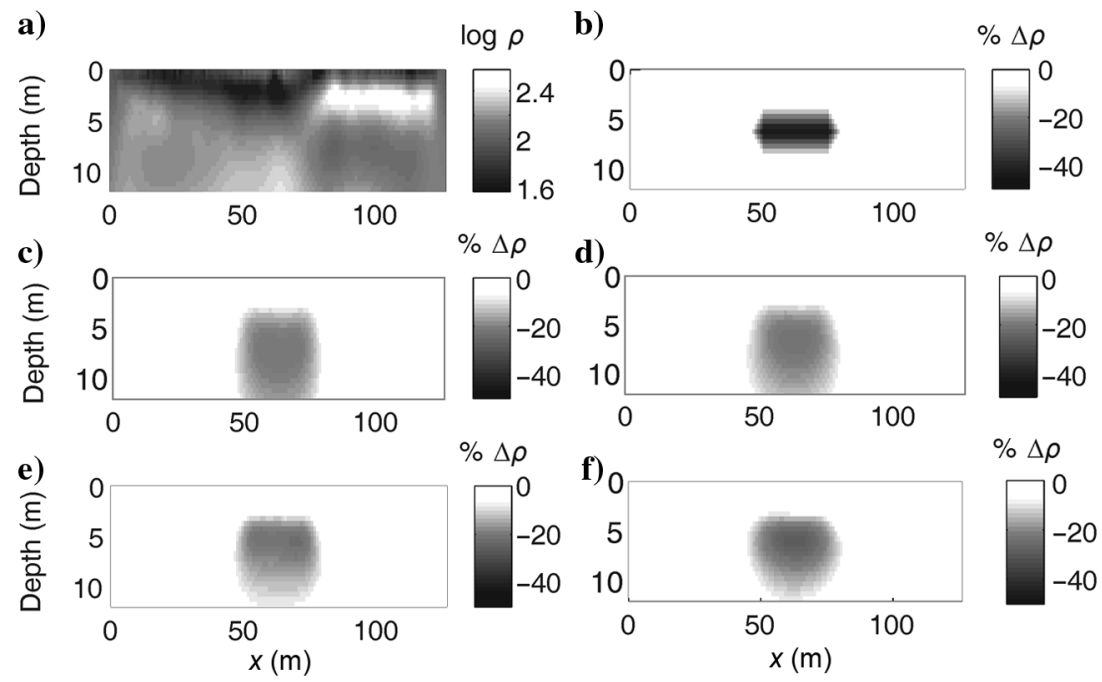

Figure 1. (a) The SCI of the background, (b) true time-lapse model, (c) time-lapse isotropic SCI, (d) time-lapse anisotropic SCI, (e) time-lapse isotropic CCI, and (f) timelapse anisotropic CCI. The CCI inversions better image the anomaly even if the horizontal range is unknown. Adding the correct anisotropy ratio slightly improves the results for SCI and CCI. corresponds to the value of the square root in equation 6 for an inverted time-lapse model with no change $\left(\rho_{\mathrm{BG}, i}^{*}=\rho_{\mathrm{TL}, i}^{*}\right.$, but $\left.\rho_{\mathrm{BG}, i} \neq \rho_{\mathrm{TL}, i}\right)$. Because $\rho_{\mathrm{BG}, i}$ might differ from $\rho_{\mathrm{BG}, i}^{*}$, equation 6 tends to penalize relative changes different from the ones observed in the true time-lapse model. A model retrieving perfectly the model changes would have a $M M_{\mathrm{TL}}$ of zero, whereas a model with no changes at all would have a $M M_{\mathrm{TL}}$ equal to one. A value more than 1 would correspond to a model with an unacceptable level of artifacts. The $M M_{\mathrm{TL}}$ is a global measure of performance averaging the result on the whole inversion section. Larger or smaller differences can be observed locally.

\section{Comparison with standard inversion}

Figure 1 shows the comparison of the time-lapse results obtained with SCI and CCI. Two different cases are presented corresponding to isotropic and anisotropic constraints. For isotropic CCI, the model parameter covariance matrix is built using a unique range of $3.2 \mathrm{~m}$, corresponding to the vertical correlation. This case intends to assess the behavior of the method when only the vertical range can be assessed, as it can be the case in practice through vertical boreholes. For the anisotropic SCI, an anisotropy ratio of 10 is used, corresponding to the ratio in $\mathrm{CCI}$ for vertical and horizontal ranges equal to 3.2 and $32 \mathrm{~m}$.

As can be expected for the isotropic SCI in time lapse (Figure 1c), the anomaly is spread almost over the whole thickness of the aquifer. The maximum change is only $-20 \%$ compared with the $-50 \%$ of the true time-lapse model. The maximum change is not detected at the correct position but approximately $1 \mathrm{~m}$ deeper. The top and lateral extension of the anomaly are relatively well-imaged. The anisotropic SCI performs slightly better with a maximum change of $-21.7 \%$ and a decrease of smoothing at depth.

The CCI results are better than SCI results. In the isotropic case, the maximum change is $-22.3 \%$, a slight improvement; the depth of the anomaly is correctly located, and its thickness better retrieved. It is spread to the bottom of the aquifer, but here a clear decrease of the amplitude of the anomaly is visible. The top and lateral extensions of the anomaly are well-imaged, similar to the SCI inversion results. For the anisotropic CCI, the amplitude of the anomaly is better estimated $(-28 \%)$.

The observations above are confirmed by the value of $M M_{\mathrm{TL}}$ for those inversions (Table 1) and by the variograms computed on the inverted tomograms for the anisotropic case (Figure 2c-2f). The $M M_{\mathrm{TL}}$ value for the anisotropic SCI is 0.73 , whereas it decreases to 0.59 for the CCI, which is a substantial improvement (24\%). The values for the isotropic cases are degraded to 0.78 for SCI and 0.66 for CCI. The ranges of the variograms for CCI (4.5 and $42 \mathrm{~m}$ for vertical and horizontal range values, respectively) are closer to those of the synthetic time-lapse model (3.2 and $32 \mathrm{~m}$, respectively), which were used to compute the model difference covariance matrix. The SCI renders an even more overestimated vertical range $(5.5 \mathrm{~m})$, whereas the horizontal range is $42 \mathrm{~m}$, too. Even though CCI does not ensure recover of the imposed correlation length, it allows 
the computing of a solution, whose vertical range is in better agreement with the true model. This reflects the fact that vertical variations in the tomograms are less controlled by the data than horizontal variations, in agreement with the well-known sensitivity/resolution characteristics of ERT surface arrays. The higher ranges in the inversion results are probably related to the general limitations of surface ERT to image an anomaly located at this depth. The differences in the sill value are related to the maximum change obtained in the tomograms.

\section{Sensitivity to the ranges}

In this section, we modify the model difference covariance matrix by dividing or multiplying horizontal and vertical ranges by two. This gives us four new inversion results to compare with the solution using the standard range from the previous section. The results are presented in Figure 3.

When the ranges are divided by two, the solutions are very close to the reference one for the standard ranges (Figure 3b). For the vertical range (Figure 3d), the anomaly is slightly thinner, but this is counterbalanced by small artifacts (increase in resistivity) near the surface that are not directly visible in the figure due to the color scale. The reason for such small changes is probably that a poorly chosen range can still be counterbalanced by information contained in the data. It means that the inversion cannot find a solution with such a small range in acceptable agreement with the data. This is also illustrated in the model misfit, which is equal to 0.61 , similar to the standard case. For the smaller horizontal range (Figure 3c), the increase in $M M_{\mathrm{TL}}$ is slightly larger and the amplitude of the maximum change in resistivity is smaller.


d)
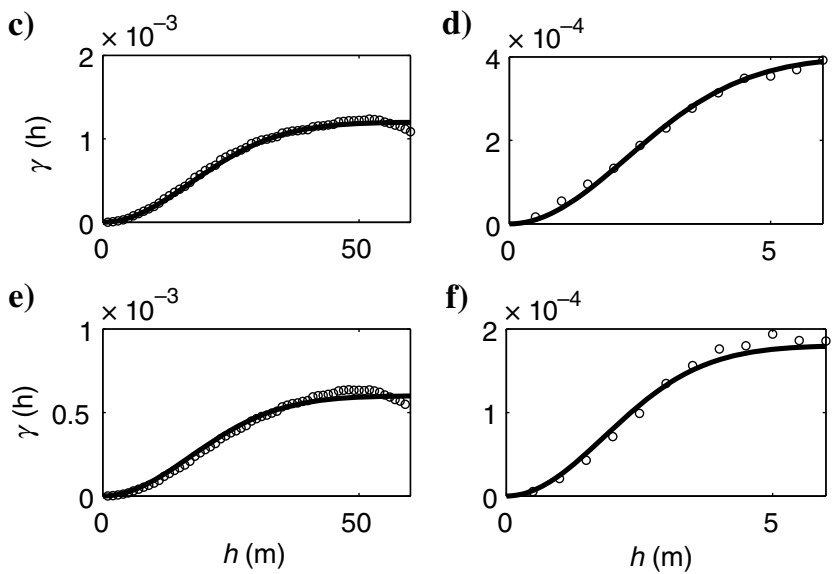

Figure 2. Experimental (circle) and modeled (line) variogram for the time-lapse synthetic model $(\mathrm{a}=$ horizontal and $\mathrm{b}=$ vertical $)$, for the SCI tomogram $(\mathrm{c}=$ horizontal and $\mathrm{d}=$ vertical $)$, and for the CCI tomogram $(e=$ horizontal and $f=$ vertical $)$ of Figure $1 d$ and 1f. The CCI renders a variogram closer to the imposed ones.
For ranges multiplied by two, the degradation of the solution compared with that for the standard ranges is more visible. A larger horizontal range (Figure $3 \mathrm{e}$ ) tends to spread the anomaly horizontally along with a decrease of the maximum amplitude of the anomaly. This is an undesirable effect because the anomaly was initially well-constrained laterally. When the vertical range is increased (Figure 3f), the anomaly is spread over an increased thickness and the position of the maximum resistivity change is now located deeper below the true one. Both model misfits are degraded.

The reason for the observed behavior probably lies in the resolution/sensitivity pattern of a surface ERT survey. The regularization operator has a strong influence in zones of low sensitivity and will impact primarily the resistivity variations in these zones. An increase in the range value will thus tend to increase smoothness in the bottom part of the model, either vertically or horizontally. However, as can be seen from Figure 1e, the horizontal correlation length seems to have less influence on the results.

As expected, the best solution is obtained for the correct ranges. However, the degradation of the results when using different ranges remains relatively small compared with the improvement brought by CCI. The four inversion results using an underestimated or overestimated range, in the horizontal and vertical directions, still perform better than SCI. This behavior has already been observed in similar studies using a model parameter covariance matrix in inverse problems (Yeh and Liu, 2000; Hansen et al., 2006; Hermans et al., 2012). It means that if one does not have access to the ranges through direct data, using a first guess of the ranges and an isotropic inversion could still be useful and yield a better result than SCI. In general, the maximum value of the range that should be considered is half of the size of the model grid in the respective direction.

Sensitivity to the depth of the anomaly

In this section, we investigate how CCI acts when the target is placed in different parts of the tomograms with various resolutions.

Table 1. Comparison of the time-lapse model misfit $\left(M M_{\mathrm{TL}}\right)$ for the inversions of the surface synthetic benchmark.

\begin{tabular}{lccccc}
\hline Case & Anomaly & Inversion & $a_{v}(\mathrm{~m})$ & $a_{h}(\mathrm{~m})$ & $M M_{\mathrm{TL}}$ \\
\hline 1 & Middle & SCIiso & - & - & 0.78 \\
2 & Middle & SCIanis & $0.1 \times a_{h}$ & - & 0.73 \\
3 & Middle & CCI & 3.2 & 3.2 & 0.66 \\
4 & Middle & CCI & 3.2 & 32 & 0.59 \\
5 & Middle & CCI & 3.2 & 16 & 0.63 \\
6 & Middle & CCI & 3.2 & 64 & 0.66 \\
7 & Middle & CCI & 1.6 & 32 & 0.61 \\
8 & Middle & CCI & 6.4 & 32 & 0.64 \\
9 & Top & SCIanis & $0.1 \times a_{h}$ & - & 0.42 \\
10 & Top & CCI & 3.2 & 32 & 0.34 \\
11 & Bottom & SCIanis & $0.1 \times a_{h}$ & - & 0.79 \\
12 & Bottom & CCI & 3.2 & 32 & 0.80 \\
\hline
\end{tabular}


In the reference case (Figure 1), the anomaly is located in the middle of the section. The size and amplitude of the anomaly as well as the ranges remain the same.

An anomaly located in the upper part of the model corresponds to a best case scenario (Figure 4a). Sensitivity and resolution are more favorable, and the decrease in resistivity should be better imaged. This is what is observed, with SCI (Figure 4b) and CCI (Figure 4c). The anomaly is correctly located vertically and horizontally. The amplitude of the anomaly is also better quantified than in the stan- dard case. However, the improvements observed in the reference case for CCI seem to be confirmed in this case too: the vertical correlation length controls the smoothing of the anomaly with depth, which yields a less spread out anomaly, and the maximum amplitude of the anomaly is closer to the true one $(-41 \%$ for CCI and $-39 \%$ for SCI). These observations are confirmed in terms of timelapse model misfits, which are equal to 0.42 and 0.34 for SCI and CCI, respectively. The relative improvement is similar to the reference case. We also computed the ranges for the inverted tomograms.
Figure 3. (a) True time-lapse model, (b) time-lapse CCI with standard ranges and inversion results with modified ranges: (c) horizontal range divided by two, (d) vertical range divided by two, (e) horizontal range multiplied by two, and (f) vertical range multiplied by two.



b)

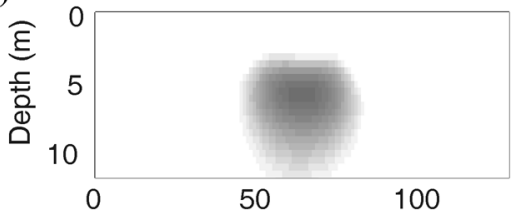

c)
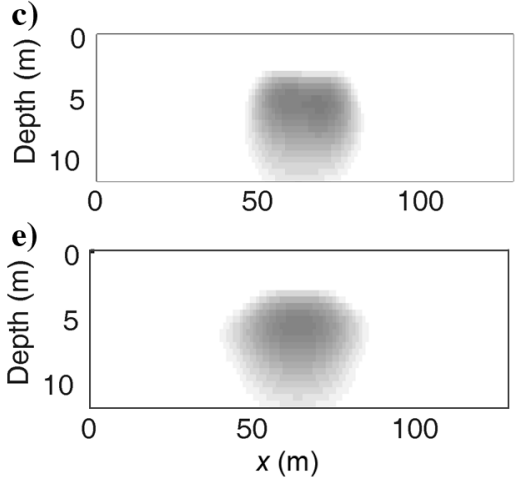

d)

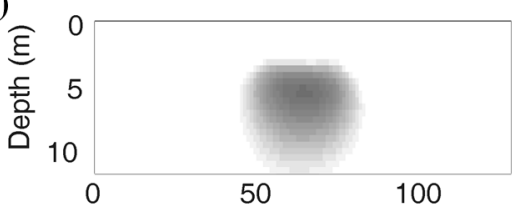

f)

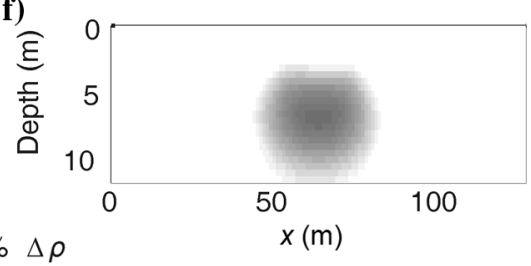

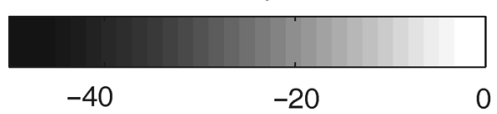

Figure 4. (a) True time-lapse model with the anomaly in the upper part of the model, (b) SCI, and (c) CCI results for the model in (a); (d) true time-lapse model with the anomaly in the bottom part of the model, (e) SCI, and (f) CCI results for the model in (d).
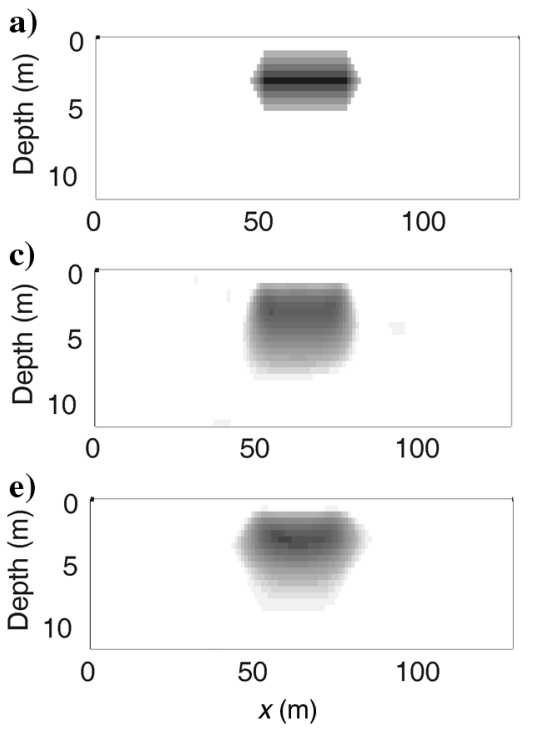

b)

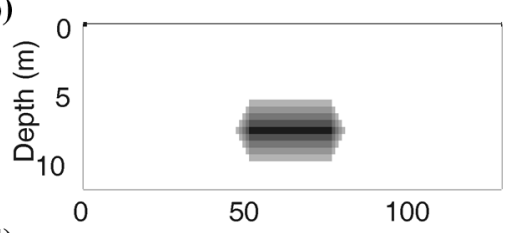

d)
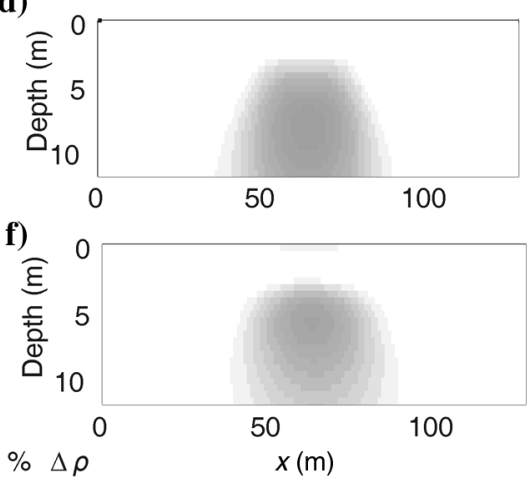

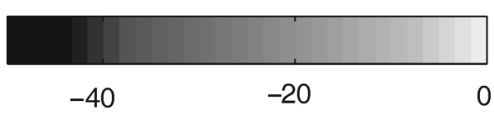


In this case, both regularizations yield a horizontal range of $35 \mathrm{~m}$, relatively close to the reference used to compute the model difference covariance matrix $(32 \mathrm{~m})$. The vertical range for the anisotropic SCI is slightly overestimated to $3.5 \mathrm{~m}$, whereas CCI manages to preserve the vertical range $(3.1 \mathrm{~m})$.

An anomaly located at larger depth is more challenging to image (Figure 4d). The sensitivity of surface ERT decreases with depth, so resistance data are less influenced by resistivity changes located in this part of the model. This is clear from Figure 4e and 4f, where the anomaly is detected, but its maximum change for both constraints is only approximately $10 \%$. A larger spacing between electrodes would have to be used to increase the depth of investigation and correctly image the anomaly in this case.

\section{Cross-borehole synthetic case}

The cross-borehole synthetic benchmark is inspired by the field case. It is a $4.5 \mathrm{~m}$ wide panel with 13 electrodes in each borehole equally spaced between 3.5 and $9.5 \mathrm{~m}$ depth. Data were simulated for a mix of bipole-bipole and dipole-dipole measurement configurations (same protocol as the field case). The true time-lapse model corresponds to an anomaly with a maximum change in resistivity of $28.7 \%$, decreasing to zero to all sides. The anomaly has the same extension in the vertical and horizontal directions, and it is best modeled with a spherical variogram with a range equal to $2.2 \mathrm{~m}$. It is located in the bottom left corner of the ERT panel (Figure 5a).
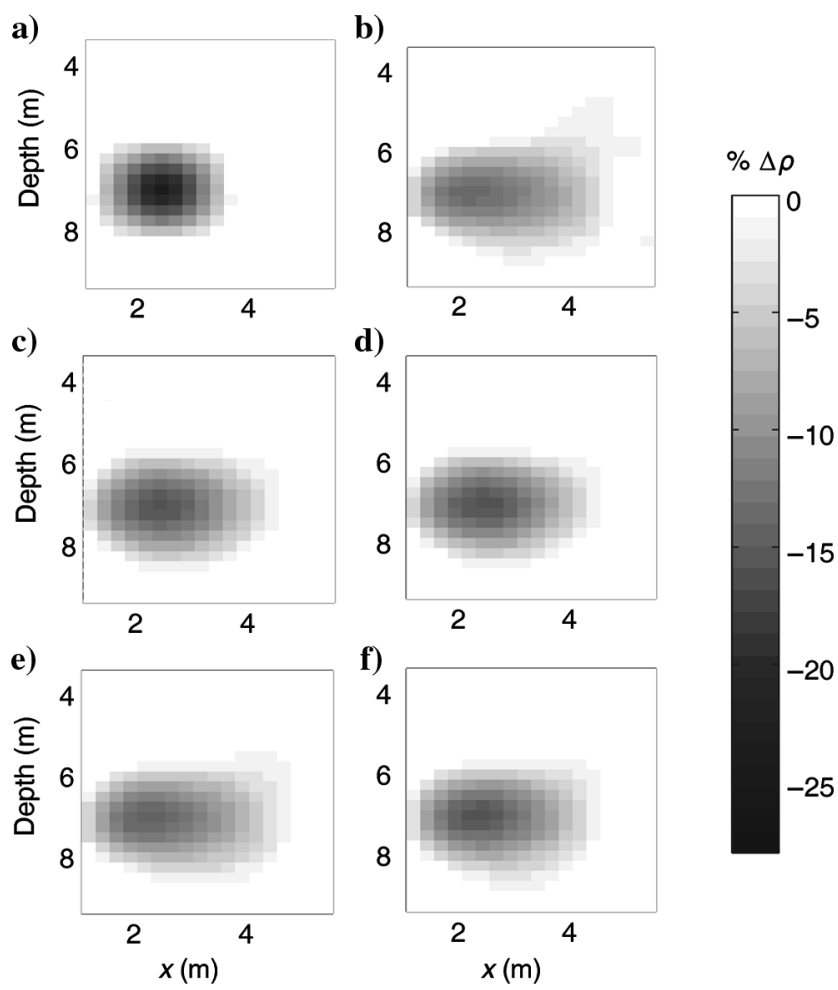

Figure 5. (a) True time-lapse model, (b) SCI, (c) CCI with spherical variogram model, (d) CCI with doubled sill, (e) CCI with exponential variogram model, and (f) CCI with Gaussian variogram model.
Data were simulated and contaminated by Gaussian noise with a standard deviation $0.5 \%$.

Figure $5 \mathrm{~b}$ and $5 \mathrm{c}$ shows the results of SCI and CCI, respectively. The latter performs better by reducing the smoothing effect toward the right and bottom parts of the section. This is confirmed by the $M M_{\mathrm{TL}}$ values (Table 2), with a decrease from 0.51 to 0.38 .

This example also illustrates how the regularization parameter scales the sill value used to calculate the covariance matrix. A solution with a value of the sill doubled (Figure 4d) renders almost identical results with a value of $\lambda$ doubled, too. Small discrepancies between the solutions arise from the line search to optimize $\lambda$, which starts from the same value for both inversions. Figure $5 \mathrm{e}$ and $5 \mathrm{f}$ shows the effect of the choice of the variogram model (exponential and Gaussian model, respectively) used to compute the covariance matrix. In practice, the choice of the variogram should be based on the available data. The Gaussian model yields results very similar to the spherical model (Figure $5 \mathrm{c}$ ) with $M M_{\mathrm{TL}}$ equal to 0.39 . With the exponential model, a degradation is observed $(0.45)$ due to the lower maximum change in the inversion result. All solutions perform better than SCI, with reduced smoothing in low-sensitivity zones (middle of the section).

\section{Summary of the synthetic studies}

In the synthetic benchmarks, we have compared SCI with our implementation of CCI for surface and crosshole time-lapse ERT. In the reference cases, where the ranges and the variogram model corresponding to the true models were used, CCI is slightly more efficient than SCI. This behavior persists when the target anomaly is located in different zones of the tomograms with sufficient sensitivity. Although the improvement observed for CCI is limited, it remains significant for a quantitative interpretation of time-lapse ERT.

For anomalies located in high-sensitivity zones, CCI seems to be able to impose the correlation length to the solution. For lower sensitivity zones, the correlation length used for regularization is not conserved in the inversion result, but an imaging improvement through reduced smoothing is still observed.

The use of CCI requires the introduction of two additional parameters in the inversion: the vertical and horizontal ranges. A sensitivity analysis on the influence of their value has shown that an error of $100 \%$ on the range value was not resulting in considerable changes of the inversion results, which remained superior to SCI.

Table 2. Comparison of the time-lapse model misfit $\left(M M_{T L}\right)$ for the inversions of the cross-borehole synthetic benchmark.

\begin{tabular}{lccc}
\hline Case & Constraint & Vario model & $M M_{\mathrm{TL}}$ \\
\hline 1 & SCI & - & 0.51 \\
2 & CCI & Spherical & 0.38 \\
3 & CCI & Spherical, sill $\times 2$ & 0.37 \\
4 & CCI & Exponential & 0.45 \\
5 & CCI & Gaussian & 0.38 \\
\hline
\end{tabular}


In our implementation, the role of the sill is counterbalanced by the regularization parameter. The variance in the computed solution is thus only dependent on the data and the assumed noise level.

The type of variogram model used to compute the covariance matrix influences the solution. However, the degradation observed for inappropriate models is limited compared with the benefits of reduced smoothing effects. In this specific study, spherical and Gaussian models yield similar results. We recommend using the type of variogram model best fitting the experimental variogram.

\section{FIELD EXPERIMENT}

CCI is now tested on a field cross-borehole ERT example. The data sets were collected during a heat-tracing experiment carried out in an alluvial aquifer. We refer readers to Hermans et al. (2015b) and Wildemeersch et al. (2014) for a detailed description of the experimental setup and the results.



Figure 6. Experimental set-up of the heat-tracing experiment.

\section{Experimental setup}

The experimental site is located in the alluvial plain of the Meuse River in Belgium. The alluvial deposits are $10 \mathrm{~m}$ thick and the water level lies $3 \mathrm{~m}$ below the surface. The saturated deposits can be distinguished in two main layers: a sandy gravel layer from 3 to $7 \mathrm{~m}$ depth and a coarse gravel layer from $7 \mathrm{~m}$ depth down to the bedrock.

The heat-tracing experiment (Figure 6) took place between an injection well and a pumping well, $20 \mathrm{~m}$ apart and parallel to the direction of groundwater flow. Water was continuously extracted from the pumping well at $30 \mathrm{~m}^{3} / \mathrm{h}$. During the first $24 \mathrm{~h}$ of the experiment, $3 \mathrm{~m}^{3} / \mathrm{h}$ were heated using a mobile water heater and reinjected in the injection well with a difference in temperature of $25^{\circ} \mathrm{C}$.

At $8 \mathrm{~m}$ distance from the injection well, a $4.5 \mathrm{~m}$ wide panel of piezometers crosses the direction of flow. The outer piezometers are screened over the whole thickness of the aquifer and were equipped during the experiment with fiber optic distributed temperature sensing (DTS, e.g., Hermans et al., 2014) and with borehole electrode cables. The 13 electrodes, spaced every $50 \mathrm{~cm}$, were located in the saturated zone from 3.5 to $9.5 \mathrm{~m}$ depth. In addition, piezometers located in the middle of the panel were equipped with temperature loggers to provide direct temperature measurements (Figure 6).

Resistance data were acquired using a combination of bipole-bipole and cross-borehole dipole-dipole configurations (Zhou and Greenhalgh, 2000) for a total of 969 measurements. We estimated the error level at each time step using reciprocal measurements and derived a common linear error model with a mixture of a two Gaussian distribution: (1) an absolute resistance error with a standard deviation of $0.002 \Omega$, and (2) a relative resistance error with a standard deviation of $0.5 \%$.

In this specific case, we used the spatially distributed temperature measurements from the DTS in one of the boreholes to derive the vertical correlation length of temperature changes (spatial resolution of $25 \mathrm{~cm}$ ). We assume that the correlation length for resistivity changes is similar given the direct relationship between temperature and electrical resistivity (Hermans et al., 2014) and valid in the entire image plane. Due to the absence of more data, we assume an isotropic constraint, i.e., equal vertical and horizontal ranges.

From the temperature distribution, we compute the variogram for each time step. An example is given in Figure 7a for the time step $30 \mathrm{~h}$ after injection. The experimental variogram is fitted using a Gaussian model with a sill equal to $4.6 \mathrm{~K}^{2}$ (related to the variance of temperature changes) and a range of $1.6 \mathrm{~m}$. The sill is changing with time, and it is increasing with the amplitude of the anomaly; however, it has little influence on the CCI results because it is balanced by the regularization factor $\lambda$ (see equation 3 ). Similarly, the range is varying with time (Figure $7 b$ ). We see that it lies between $0.9 \mathrm{~m}$ for late time steps and $1.9 \mathrm{~m}$ approximately $25 \mathrm{~h}$ after the beginning of the experiment.

\section{Results}

The data for each time step were inverted with the difference inversion scheme using SCI and CCI. For the latter, the corresponding range obtained from the independent temperature data
Figure 7. (a) The experimental variogram for the time step $30 \mathrm{~h}$ after beginning of injection and its description by a Gaussian model with a range equal to $1.6 \mathrm{~m}$. (b) The range of the fitted variogram model evolves with time, with a minimum range of $0.9 \mathrm{~m}$ and a maximum range of $1.9 \mathrm{~m}$.

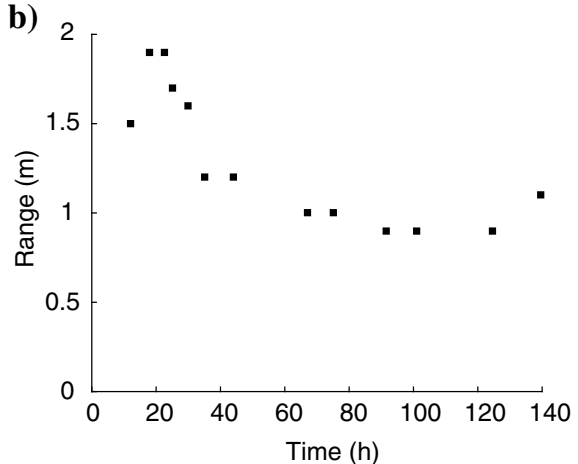


was used (Figure 7b). A data set acquired before injection was used as the background for all subsequent time steps. The background model was obtained with SCI (Figure 5 in Hermans et al., 2015b).

Figure 8 shows six selected time steps comparing the two regularization methods. Qualitatively, we see that the results are coherent for both methods. The anomaly is first growing in amplitude; it reaches a maximum and decreases slowly toward its initial state. Although the heated water was injected over the whole thickness of the aquifer, it preferentially flows in the deeper part of the aquifer. This can be explained by the contrast in hydraulic conductivity between the sandy gravel and the coarse gravel layers, which is approximately two orders of magnitude.

The anomaly is divided laterally into two different parts. The one with higher contrast is located near the first ERT borehole, and the second one is closer to the second borehole. Thus, we have a decrease in the resistivity anomaly (and in temperature) in the middle of the bottom part of the section, where the sensitivity is lowest (Figure 6 in Hermans et al. [2015b] for the specific sensitivity pat- tern of the experiment). This shape of the anomaly is necessary to explain the data, and it is confirmed by direct temperature measurements in control piezometers between ERT boreholes (Wildemeersch et al., 2014; Hermans et al., 2015b). It is probably related to a low hydraulic conductivity (clay lens) zone between the injection well and the ERT panel.

In the zones of lower sensitivity, more significant differences can be observed between the inversion results. We clearly see that SCI tends to produce a very smooth image with resistivity changes spreading laterally and in depth, as it was observed in the synthetic case. Although showing a minimum laterally, the two zones of maximum resistivity changes tend to be connected in the middle of the section. Similarly, the anomaly is spread toward the bottom of the aquifer. This is less the case for CCI, in which the resistivity changes are more concentrated in two anomalies with slightly larger amplitudes in terms of percentage changes.

The comparison with direct measurements available during the experiment confirms that the two solutions differ mainly in the
$\mathrm{SCl}$

$\mathrm{CCl}$

$18 \mathrm{~h}$


$25 \mathrm{~h}$
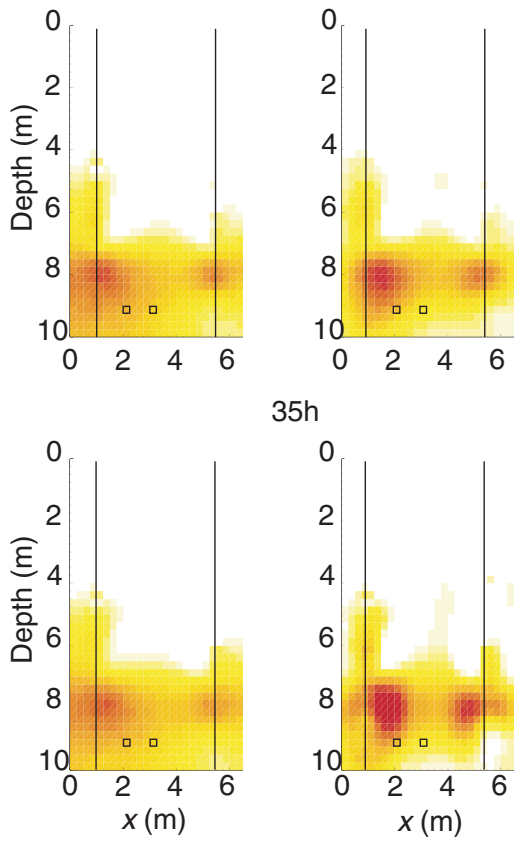

$\% \Delta \rho$

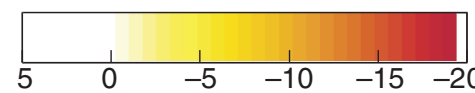

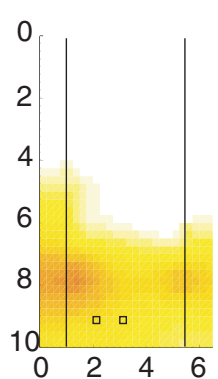

$44 h$

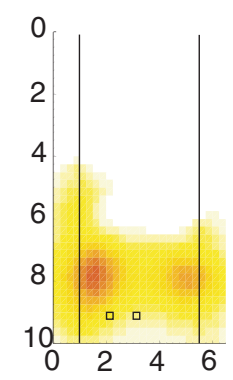

$67 \mathrm{~h}$
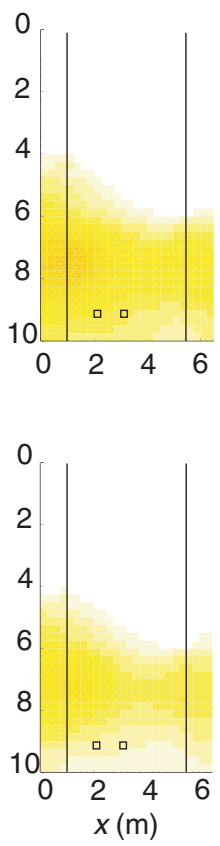
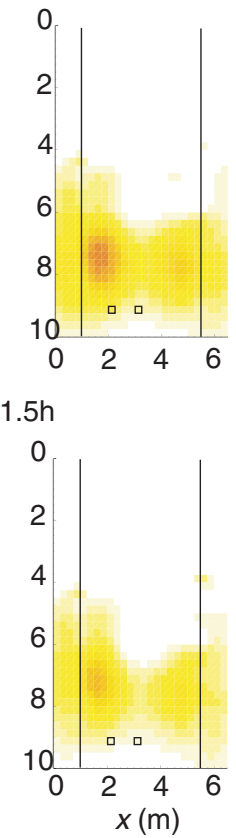

Figure 8. Comparison of SCI and CCI results for various time steps during the heat-tracing experiment. 




Figure 9. Comparison of SCI and CCI results with DTS measurements in the second ERT borehole $30 \mathrm{~h}$ after the beginning of injection. The inversion results are relatively similar because the borehole is located in a high-sensitivity zone of the tomogram.

lower sensitivity zone. For this comparison, resistivity changes were converted into temperature variations using a calibrated relationship (Hermans et al., 2015b). Figure 9 shows the comparison of DTS measurements with the ERT inversion results in the second borehole. The sensitivity of crosshole ERT is highest in the vicinity of the boreholes, and we see that here the inversion results are relatively similar for both constraints.

Two temperature loggers are available in the bottom part of the aquifer (Figures 6 and 8). They are located at different positions between the ERT boreholes. We extracted the ERT-derived temperatures at the position of these loggers to compare them with the breakthrough curves measured directly (Figure 10). For D1, which is only $1 \mathrm{~m}$ from one of the boreholes, the two ERT curves are relatively similar. They recover fairly well the maximum of the curve but overestimate the tailing part. This can be related to oversmoothing or 3D effects (Hermans et al., 2015b). However, in this part, the temperature obtained from $\mathrm{CCI}$ is closer to the one measured directly in the borehole.

The second temperature logger D2 is located almost in the middle of the panel, where the sensitivity is very low. This means that the regularization is dominant in this zone. Here, the improvement brought by CCI is clear. The temperatures of the CCI breakthrough curve are almost identical to the direct measurements for the tailing part of the curve. In addition, the amplitude of the maximum change is better retrieved, with temperatures only slightly underestimated.
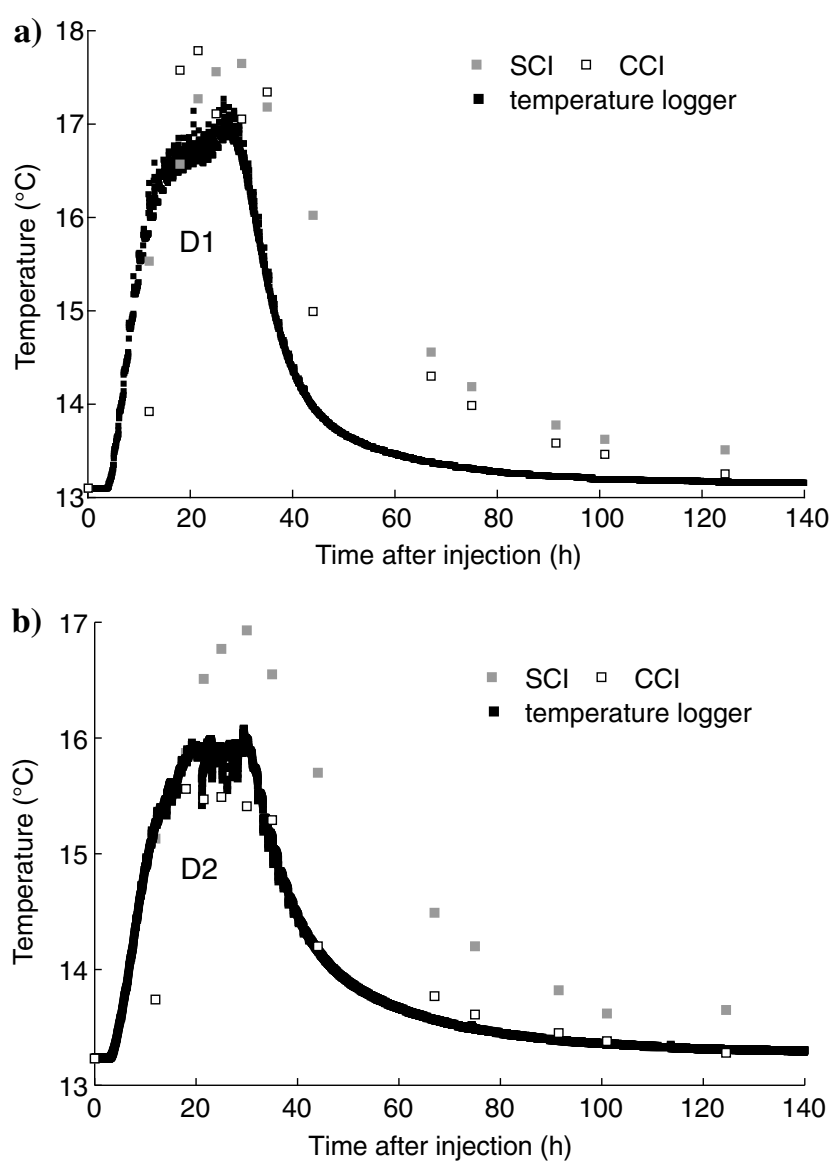

Figure 10. Comparison of SCI and CCI results with direct measurements of the temperature loggers at the locations (a) D1 and (b) D2 (see Figures 6 and 8 ). The tailing parts of the breakthrough curves are better imaged with CCI, mainly in zones of low sensitivity (D2).

The overestimation of temperature in this part of the aquifer with SCI is related to over-smoothing of the anomaly. These observations are coherent with the results of the synthetic benchmark, which stressed that low-sensitivity zones are more impacted by the choice of the regularization. This example illustrates how slight changes in the recovered resistivity change distribution might significantly improve quantitative interpretation.

\section{CONCLUSIONS}

Recent advances in geophysical imaging have broadened the range of applications and scales to which time-lapse ERT can be successfully applied. We can therefore expect in the near future a relatively high growth rate for time-lapse ERT applications, in research and in the industry. Many tasks remain to be done to improve the imaging capabilities of ERT in those contexts regarding noise analysis, data integration, and regularization.

For now, most studies have focused on the time aspect of the time-lapse inverse problem, trying to simultaneously invert all data sets in a true 4D model parameter space, or on model functionals leading to sharp contrasts, such as the minimum-gradient support. In this study, we instead focused on the spatial regularization of individual steps of the time-lapse ERT inverse problem. We propose 
to use a covariance-based constraint of model differences to replace the standard smoothness constraint operator in the difference inversion scheme.

For surface ERT, we have shown with a synthetic benchmark that this kind of regularization was more successful than the smoothness constraint to image the gradual changes in resistivity of our reference model. The covariance-based regularization performed better than the smoothness constraint in high- and low-sensitivity/resolution parts of the tomogram. The center of the mass of the anomaly was systematically better retrieved, and the smoothing effect of the inversion was reduced to more acceptable levels. The ranges computed from the inverted tomograms are more in agreement with the true model.

Our implementation is based on the introduction of two additional parameters: the vertical and horizontal ranges of the model difference covariance matrix. However, a sensitivity analysis has shown that uncertainty in the computation of the ranges does not have a significant influence on the inversion results. A small degradation was observed, but the global results remained better than the standard smoothness-constrained solution. This means that if few data are available and the uncertainty on the ranges is high, it may still be worth using a CCI instead of the SCI.

The methodology was also applied to crosshole ERT data for a synthetic and a field case, where a heat-tracing experiment was monitored. The synthetic benchmark has shown the robustness of the method regarding the type of variogram model and the value of the sill. The observations made for the synthetic benchmark were confirmed by the comparison of the inversion results of the field case with independent direct measurements. Except for the first time step for which the smoothness constraint might be locally more accurate, the CCI globally yields more consistent results. Most significant imaging improvements were observed in the zone of lowest sensitivity of the tomograms, where the use of the CCI enabled retrieving the breakthrough curve more accurately measured with a temperature logger. This improvement is due to the reduction of the smoothing effect in the inversion process. In highly sensitive zones, both inversions yield relatively similar results.

Standard SCI can be improved by imposing an adequate anisotropic smoothing. However, when sufficient data are available, we recommend using the CCI to improve imaging results. The latter acts as a smoothing operator but its correlation length can be controlled and based on independent data, such as direct measurements in boreholes. Even in the absence of sufficient spatially distributed data, a trial and error approach can be used to optimize the ranges used in the inversion by comparing the results with local observations. Although improvement might remain relatively limited, it might still be significant for a quantitative interpretation of timelapse ERT.

The CCI applied here in the spatial dimensions can be extended to the time dimension using a combined spatio-temporal variogram model. This would open up the use of covariance-based constraints in $4 \mathrm{D}$ inversion schemes addressing the issue of combined spatial and temporal regularizations. This should be investigated in future work.

\section{ACKNOWLEDGMENT}

We thank the F.R.S.-FNRS for their financial support of T. Hermans as a postdoctoral researcher. We also thank three anonymous reviewers for their constructive comments that helped in improving the quality of the manuscript.

\section{REFERENCES}

Ajo-Franklin, J. B., B. J. Minsley, and T. M. Daley, 2007, Applying compactness constraints to differential traveltime tomography: Geophysics, 72, no. 4, R67-R75, doi: 10.1190/1.2742496.

Arato, A., J. Boaga, C. Comina, M. De Seta, E. Di Sipio, A. Galgaro, N. Giordano, and G. Mandrone, 2015, Geophysical monitoring for shallow geothermal applications - Two Italian case histories: First Break, 33, 75 79

Audebert, M., R. Clément, N. Touze-Foltz, T. Günther, S. Moreau, and C. Duquennoi, 2014, Time-lapse ERT interpretation methodology for leachate injection monitoring based on multiple inversions and a clustering strategy (MICS): Journal of Applied Geophysics, 111, 320-333.

Binley, A., S. S. Hubbard, J. A. Huisman, A. Revil, D. A. Robinson, K. Singha, and L. D. Slater, 2015, The emergence of hydrogeophysics for improved understanding of subsurface processes over multiple scales: Water Resources Research, 51, 3837-3866, doi: 10.1002/2015WR017016.

Binley, A., P. Winship, L. J. West, M. Pokar, and R. Middleton, 2002, Seasonal variation of moisture content in unsaturated sandstone inferred from borehole radar and resistivity profiles: Journal of Hydrology, 267, 160 172.

Blaschek, R., A. Hördt, and A. Kemna, 2008, A new sensitivity-controlled focusing regularization scheme for the inversion of induced polarization based on the minimum gradient support: Geophysics, 73, no. 2, F45-F54 doi: $10.1190 / 1.2824820$

Carrigan, C. R., X. Yang, D. J. LaBrecque, D. Larsen, D. Freeman, A. L. Ramirez, W. Daily, R. Aines, R. Newmark, J. Friedman, and S. Hovorka 2013, Electrical resistance tomographic monitoring of $\mathrm{CO}_{2}$ movement in deep geologic reservoirs: International Journal of Greenhouse Gas Control, 18, 401-408.

Caterina, D., T. Hermans, and F. Nguyen, 2014, Case studies of prior information in electrical resistivity tomography: Comparison of different approaches: Near Surface Geophysics, 12, 451-465, doi: 10.3997/ 1873-0604.2013070.

Chasseriau, P., and M. Chouteau, 2003, 3D gravity inversion using a model of parameter covariance: Journal of Applied Geophysics, 52, 59-74.

Coscia, I., S. A. Greenhalgh, N. Linde, J. Doetsch, L. Marescot, T. Gu, T Vogt, and A. Green, 2011, 3D crosshole ERT for aquifer characterization and monitoring of infiltrating river water: Geophysics, 76, no. 2, G49G59, doi: 10.1190/1.3553003

Day-Lewis, F. D., J. M. Harris, and S. M. Gorelick, 2002, Time-lapse inversion of crosswell radar data: Geophysics, 67, 1740-1752, doi: 10 $.1190 / 1.1527075$.

de Groot-Hedlin, C., and S. Constable, 1990, Occam's inversion to generate smooth, two dimensional models from magnetotelluric data: Geophysics, 55, 1613-1624, doi: 10.1190/1.1442813.

Doetsch, J., N. Linde, and A. Binley, 2010, Structural joint inversion of timelapse crosshole ERT and GPR traveltime data: Geophysical research Letters, 37, L24404.

Doetsch, J., N. Linde, M. Pessognelli, A. G. Green, and T. Günther, 2012a, Constraining 3-D electrical resistance tomography with GPR reflection data for improved aquifer characterization: Journal of Applied Geophysics, 78, 68-76, doi: 10.1016/j.jappgeo.2011.04.008.

Doetsch, J., N. Linde, T. Vogt, A. Binley, and A. G. Green, 2012b, Imaging and quantifying salt-tracer transport in a riparian groundwater system by means of 3D ERT monitoring: Geophysics, 77, no. 5, B207-B218, doi: 10 $.1190 / \mathrm{geo} 2012-0046.1$.

Farquharson, C. G., and D. W. Oldenburg, 1998, Non-linear inversion using general measures of data misfit and model structure: Geophysical Journal International, 134, 213-227.

Fiandaca, G., J. Doetsch, G. Vignoli, and E. Auken, 2015, Generalized focusing time-lapse changes with applications to direct current and time-domain induced polarization inversions: Geophysical Journal International, 203, 1101-1112.

Franklin, J. N., 1970, Well-posed stochastic extensions of ill-posed linear problems: Journal of Mathematical Analysis and Applications, 31, 682-716.

Hansen, T. M., A. G. Journel, A. Tarantola, and K. Mosegaard, 2006, Linear inverse Gaussian theory and geostatistics: Geophysics, 71, no. 6, R101R111, doi: 10.1190/1.2345195.

Hermans, T., F. Nguyen, T. Robert, and A. Revil, 2014, Geophysical methods for monitoring temperature changes in shallow low enthalpy geothermal systems: Energies, 7, 5093-5118.

Hermans, T., F. Nguyen, and J. Caers, 2015a, Uncertainty in training imagebased inversion of hydraulic head data constrained to ERT data: Workflow 
and case study: Water resources Research, 51, 5332-5352, doi: 10.1002/ 2014WR016460

Hermans, T., A. Vandenbohede, L. Lebbe, R. Martin, A. Kemna, J. Beaujean, and F. Nguyen, 2012, Imaging artificial salt water infiltration using electrical resistivity tomography constrained by geostatistical data: Journal of Hydrology, 438-439, 168-180, doi: 10.1016/j.jhydrol.2012.03.021.

Hermans, T., S. Wildemeersch, P. Jamin, P. Orban, S. Brouyère, A. Dassargues, and F. Nguyen, 2015b, Quantitative temperature monitoring of a heat tracing experiment using cross-borehole ERT: Geothermics, 53, 14-26.

Hinnel, A. C., T. P. A. Ferre, J. A. Vrugt, J. A. Huisman, S. Moysey, J. Rings, and M. Kowalsky, 2011, Improved extraction of hydrologic information from geophysical data through coupled hydrogeophysical inversion: Water resources Research, 46, W00D40.

Holliger, K., J. Tronicke, H. Paasche, and B. Dafflon, 2008, Quantitative integration of hydrogeophysical and hydrological data: Geostatistical approaches, in C. Darnault, ed., Overexploitation and contamination of shared groundwater resources: Springer Netherlands, 67-82.

Irving, J., and K. Singha, 2010, Stochastic inversion of tracer test and electrical geophysical data to estimate hydraulic conductivities: Water resources Research, 46, W11514.

Isaaks, E. H., and R. M. Srivastava, 1989, An introduction to applied geostatistics: Oxford University Press, 561.

Johnson, T. C., P. S. Routh, T. Clemo, W. Barrash, and W. P. Clement, 2007, Incorporating geostatistical constraints in nonlinear inversion problems: Water resources Research, 43, W10422.

Johnson, T. C., R. J. Versteeg, M. Rockhold, L. D. Slater, D. Ntarlagiannis, W. J. Greenwood, and J. Zachara, 2012, Characterization of a contaminated wellfield using $3 \mathrm{D}$ electrical resistivity tomography implemented with geostatistical, discontinuous boundary, and known conductivity constraints: Geophysics, 77, no. 6, EN85-EN96, doi: 10.1190/geo2012-0121.1.

Kaipio, J. P., V. Kolehmainen, M. Vauhkonen, and E. Somersalo, 1999, Inverse problems with structural prior information: Inverse Problems, 15 713-729.

Karaoulis, M. C., J. H. Kim, and P. I. Tsourlos, 2011, 4D active time constrained resistivity inversion: Journal of Applied Geophysics, 73, 25-34, doi: 10.1016/j.jappgeo.2010.11.002.

Kemna, A., 2000, Tomographic inversion of complex resistivity: Theory and application: Ph.D. thesis, Ruhr-University of Bochum.

Kemna, A., J. Vanderborght, B. Kulessa, and H. Vereecken, 2002, Imaging and characterization of subsurface solute transport using electrical resistivity tomography (ERT) and equivalent transport models: Journal of Hydrology, 267, 125-146.

Kim, J. H., M. J. Yi, S. G. Park, and J. G. Kim, 2009, 4-D inversion of DC resistivity monitoring data acquired over a dynamically changing earth model: Journal of Applied Geophysics, $\mathbf{6 8}, 522-532$.

Koestel, J., A. Kemna, M. Javaux, A. Binley, and H. Vereecken, 2008, Quantitative imaging of solute transport in an unsaturated and undisturbed soil monolith with 3D ERT and TDR: Water Resources Research, 44, W12411, doi: 10.1029/2007WR006755.

Krautblatter, M., S. Verleysdonk, A. Flores Orozco, and A. Kemna, 2010 Temperature-calibrated imaging of seasonal changes in permafrost rock walls by quantitative electrical resistivity tomography (Zugspitze, German/Austrian Alps): Journal of Geophysical Research, 115, F02003, doi: 10.1029/2008JF001209.

LaBrecque, D. J., and X. Yang, 2001, Difference inversion of ERT data: A fast inversion method for 3-D in-situ monitoring: Journal of Environmental and Engineering Geophysics, 6, 83-90.
Linde, N., A. Binley, A. Tryggvason, L. B. Pedersen, and A. Revil, 2006, Improved hydrogeophysical characterization using joint inversion of cross-hole electrical resistance and ground-penetrating radar traveltime data: Water resources Research, 42, W12404, doi: 10.1029/ 2006WR0055131.

Maurer, H., K. Holliger, and D. Boerner, 1998, Stochastic regularization: Smoothness or similarity: Geophysical Research Letters, 25, 2889-2892.

Michalak, A. M., and S. Shlomi, 2007, A geostatistical data assimilation approach for estimating groundwater plume distributions from multiple monitoring events, in D. W. Hyndman, F. D. Day-Lewis, and K. Singha, eds., Subsurface hydrology, data integration for properties and processes: American Geophysical Union, Geophysical Monograph Series 171, 7388.

Nguyen, F., T. Robert, A. Kemna, and T. Hermans, 2016, Data-driven selection of the minimum gradient support parameter in time-lapse focused electrical imaging: Geophysics, 81, no. 1, A1-A5, doi: 10.1190/ geo2015-0226.1.

Oware, E., S. Moysey, and T. Khan, 2013, Physically based regularizatiom of hydrogeophysical inverse problems for improved imaging of processdriven systems: Water Resources Research, 49, 6238-6247.

Pidlisecky, A., E. Haber, and R. Knigth, 2007, RESINV3D: A 3D resistivity inversion package: Geophysics, 72, no. 2, H1-H10, doi: 10.1190/1 .2402499 .

Portniaguine, O., and M. S. Zhdanov, 1999, Focusing geophysical inversion images: Geophysics, 64, 874-887, doi: 10.1190/1.1444596.

Robert, T., D. Caterina, J. Deceuster, O. Kaufmann, and F. Nguyen, 2012, A salt tracer test monitored with surface ERT to detect preferential flow and transport paths in fractured/karstified limestones: Geophysics, 77, no. 2, B55-B67, doi: 10.1190/geo2011-0313.1.

Rosas Carbajal, M., N. Linde, and T. Kalscheuer, 2012, Focused time-lapse inversion of radio and audio magnetotelluric data: Journal of Applied Geophysics, 84, 29-38.

Singha, K., F. D. Day-Lewis, T. Johnson, and L. D. Slater, 2015, Advances in interpretation of subsurface processes with time-lapse imaging: Hydrological Processes, 29, 1549-1576.

Slater, L., A. M. Binley, W. Daily, and R. Johnson, 2000, Cross-hole electrical imaging of a controlled saline tracer injection: Journal of Applied Geophysics, 44, 85-102.

Supper, R., D. Ottowitz, B. Jochum, A. Römer, S. Pfeiler, S. Kauer, M. Keushnig, and A. Ita, 2014, Geoelctrical monitoring of frozen ground and permafrost in alpine areas: Field studies and considerations towards an improved measuring technology: Near Surface Geophysics, 12, 93115.

Tarantola, A., and B. Valette, 1982, Generalized non-linear problems solved using the least-squares criterion: Review of Geophysics and Space Physics, 20, 219-232.

Wildemeersch, S., P. Jamin, P. Orban, T. Hermans, F. Nguyen, S. Brouyère, and A. Dassargues, 2014, Coupling heat and chemical tracer experiments for estimating heat transfer parameters in shallow alluvial aquifers: Journal of Contaminant hydrology, 169, 90-99.

Yeh, T.-C. J., and S. Liu, 2000, Hydraulic tomography: Development of a new aquifer test method: Water Resources Research, 36, 2095-2105.

Zhou, B., and S. A. Greenhalgh, 2000, Cross-hole resistivity tomography using different electrode configurations: Geophysical Prospecting, 48, 887-912. 